Work on A supergiants is now underway opening up the prospect of spectroscopic distance determinations over cosmologically significant ranges with the coming generation of $8 \mathrm{~m}$ ground-based telescopes.

In constructing the model atoms and calculating the atomic data needed, it soon becomes apparent that one of the most serious deficiencies is the small number of energy levels and wavelengths available for many of the iron group ions. These are important for spectral synthesis. Moderately accurate collisional data, partial photoionization cross sections and many more line broadening data would also be most welcome.

Anderson, L.A., 1989, ApJ 339, 558

Auer, L.H., Heasley, J.N., 1976, ApJ 205, 165

Becker, S.R., Butler, K., 1992, A\&A 265, 647

Becker, S.R., Butler, K., 1994a, A\&A in press

Becker, S.R., Butler, K., 1994b, A\&A in press

Becker, S.R., Butler, K., 1994c, A\&A submitted

Dreizler, S. and Werner, K., 1993, A\&A 278, 199

Grigsby, J.A., Morrison, N.D. and Anderson, L.S., 1992, ApJS 78, 205

Kudritzki, R.P. and Hummer, D.G., 1990, Ann, Rev, Astron. and Astrophys. 28, 030

Kudritzki, R.P., Lennon, D.H. and Puls, J., 1995, in "Science with the VLT", ESO Conf., in press

Kurucz, R.L., 1988, Transactions of the Internal Astronomical Union, M. McNally, ed., Dordrecht: Kluwer, XXB, 168

Kurucz, R.L., 1991, in Stellar Atmospheres: Beyond Classical Models, NATO

ASI series C, Vol 341, eds L. Crivalleri, I. Hubeny and D.G. Hummer, Kluwer, Dordrecht, p.441 Pauldrach, A.W.A., Kudritzki, R.P., Puls, J., Butler, K. and Hunsinger, 1994, A\&A 283, 525

Werner, K. and Husfeld, D., 1985, A\&A 148, 417

\title{
WHAT NEBULAR OBSERVATIONS AND PHOTOIONIZATION MODELS CAN TELL US ABOUT ATMOSPHERES OF HOT STARS
}

\author{
ROBERT H. RUBIN ${ }^{1,2}$, DIETMAR KUNZE ${ }^{3}$, AND TETSUO YAMAMOTO
}

${ }^{\prime}$ NASA-Ames Research Center, MS 245-6, Moffett Field, CA 94035-1000

${ }^{2}$ Orion Enterprises, 2135 Woodleaf Way, Mountain View, CA 94040

${ }^{3}$ Max Planck Institut, für Astronomy, D-81679 München, Germany

'Institut fur Astronomie und Astrophysik der Universität München, Scheinerstr. 1, 81679 München, Germany

A major purpose of this paper is to emphasize to the stellar atmosphere community 
that there are tests of stellar atmospheres modeling that result from observations of nebulae associated with hot stars, and which are usually overlooked. We illustrate this point with a specific example where there is a persistent problem matching certain observed nebular emission line fluxes with photoionization models of $\mathrm{H}$ il regions. Evidence is presented for what is called the [ $\mathrm{Ne} \mathrm{III]} \mathrm{problem} \mathrm{-} \mathrm{that} \mathrm{is,} \mathrm{the} \mathrm{H}$ II region models predict significantly less flux in the [Ne III] lines than is measured. The predicted nebular fluxes are affected not only by the representation of the stellar emergent ionizing spectra but also by the prodigious set of atomic data, that is an input ingredient in such calculations. However, it is argued that difficulties with matching [Ne III] fluxes are most likely due to the use of LTE stellar atmosphere models to represent the hot stars ionizing the observed $\mathrm{H}$ II regions.

Post-meeting calculations utilizing atmospheres by Kunze that do not assume LTE permit us to directly test the above conjecture. When these new atmospheres are used in the model nebula code, there are significantly enhanced [Ne III] fluxes compared with those run using the LTE atmospheres. When we consider a reasonable range of nebular parameters as well as reasonable expectations for Kunze models with effective temperature $T_{\text {eff }}=35000$ $40000 \mathrm{~K}$, the [Ne III] problem is likely resolved. As an additional benefit of the model nebula calculations with Kunze non-LTE and Kurucz LTE atmospheres, we are able to also examine several criteria that have been used to estimate a characteristic $\mathrm{T}_{\text {eff }}$ for $\mathrm{H}$ II regions. Three indicators of "ionization" based on measured line ratios are examined. Two are from far-infrared data: [O III] $52 \mu \mathrm{m} /[\mathrm{S}$ III] $33 \mu \mathrm{m}$ and $[\mathrm{N} \mathrm{III]} 57 \mu \mathrm{m} /[\mathrm{N} \mathrm{II}] 122 \mu \mathrm{m}$; the other is the ratio of adjacent radio recombination lines of helium and hydrogen. For a given line ratio in any of these three cases, the $T_{\text {eff }}$ inferred will be lower for the non-LTE stellar atmosphere compared with the LTE one. Likewise, a non-LTE stellar atmosphere will be more highly ionized than the corresponding (same $\mathrm{T}_{\text {eff }}$, surface gravity, and composition) LTE atmosphere.

\section{OPACITIES IN STRONG MAGNETIC FIELDS}

\section{DAYAL T. WICKRAMASINGHE}

The Astrophysical Theory Centre, Australian National University, Canberra ACT 2601, Australia

White dwarfs are one of the most readily studied end products of stellar evolution. Their observed properties have provided and continue to provide important constraints for the theory of stellar evolution. Likewise, a study of magnetism in white dwarfs provides unique insights into the origin and evolution of magnetic fields in stars.

Spectacular progress has been made on the specific problem of the structure of the 